CLINICAL STUDY

\title{
Effects of standard glucocorticoid replacement therapies on subjective well-being: a randomized, double-blind, crossover study in patients with secondary adrenal insufficiency
}

\author{
Sven Benson $^{1, *}$, Pia Neumann ${ }^{2, *}$, Nicole Unger ${ }^{2}$, Manfred Schedlowski $^{1}$, Klaus Mann ${ }^{2}$, Sigrid Elsenbruch $^{1}$ and $^{2}$ \\ Stephan Petersenn ${ }^{2}$ \\ ${ }^{1}$ Institute of Medical Psychology and Behavioural Immunobiology and ${ }^{2}$ Department of Endocrinology, Division of Laboratory Research, Medical Center, \\ University Hospital Essen, University of Duisburg-Essen, Hufelandstraße 55, 45122 Essen, Germany
}

(Correspondence should be addressed to S Petersenn; Email: stephan.petersenn@endoc-med.de)

*(S Benson and P Neumann contributed equally to this work)

\begin{abstract}
Objective: For secondary adrenal insufficiency (SAI), established biochemical parameters for dosage control are lacking and no optimal substitution dosage and daily distribution have been determined yet. Therefore, in clinical practice, the individual total dose is often adjusted based on patients' subjective well-being.

Design: Effects of three standard glucocorticoid replacement regimens on psychological variables were assessed in patients with SAI based on a randomized double-blind study design.

Methods: SAI patients $(n=18)$ were treated with three different established glucocorticoid replacement regimens in a randomized, double-blind, crossover study (treatment $\mathrm{A}$, hydrocortisone $10 \mathrm{mg}$-placebo$5 \mathrm{mg}$-placebo; treatment $\mathrm{B}$, hydrocortisone $10 \mathrm{mg}-5 \mathrm{mg}$-placebo- $5 \mathrm{mg}$; and treatment $\mathrm{C}$, prednisone $5 \mathrm{mg}$-placebo-placebo-placebo). Following each 4-week replacement regimen, quality of life (SF-36) and emotional distress (brief symptom inventory (BSI)) were assessed along with diurnal changes in current well-being (Bf-S) and alertness (Stanford Sleepiness Scale (SSS)) using validated questionnaires, and additionally compared with patient (patients with pituitary disease and adrenal sufficiency) and healthy control groups.

Results: SAI patients showed improvements in physical quality of life (i.e. SF-36 physical function, $P<0.05$; physical role function, $P<0.05$ ) and current well-being (at $1800 \mathrm{~h}, P<0.05$ ) under treatment A (hydrocortisone 10-0-5-0 mg) compared with the other replacement regimens. Quality of life and current well-being were significantly impaired compared with healthy controls but did not differ from patient controls.

Conclusions: Although the observed improvements in psychological parameters were comparatively small, our results indicate beneficial effects of a 10-0-5-0 mg hydrocortisone replacement regimen. Nevertheless, treatment effects were insufficient to restore subjective health compared with healthy controls, indicating the need for improved replacement regimens and supportive psychosocial interventions in SAI patients.
\end{abstract}

European Journal of Endocrinology 167 679-685

\section{Introduction}

Adrenal insufficiency (AI) has a major impact on quality of life and subjective health $(1,2)$ and increases the risk for affective disorders (3). Hydrocortisone (HC) and the synthetic steroid prednisone are the most commonly used compounds for substitution therapy. As established biochemical parameters for dosage control are lacking (4), the individual total dose is adjusted based on subjective parameters such as fatigue or quality of life $(1,4)$.

Previous studies found little differences between standard replacement regimens and their effects on subjective health and quality of life $(5,6,7,8,9)$; however, many of these studies were cross-sectional $(7,8,9)$. Other studies investigated less physiological HC regimes, suggesting an advantage of imitating the circadian rhythm of cortisol secretion $(10,11)$. Experimental crossover studies offer the advantage to control for interindividual variation as each patient is treated with different replacement regimens and is hence his or her own control. However, such studies are generally scarce and included only a very small number of patients (i.e. 7-14 patients). The only study in patients with secondary AI (SAI) thus far (6) could not detect an effect of different $\mathrm{HC}$ dosages (i.e. 15, 20, and $30 \mathrm{mg} /$ day) on subjective well-being. This may, 
however, be attributable to the small sample size of nine SAI patients and/or the comparatively short treatment period of 2 weeks. Furthermore, differences in timing of the doses were not studied. The remaining studies assessed only patients with primary AI $(2,10,11)$ or did not differentiate between patients with primary AI and SAI (5). Hence, the aim of this study was to analyze the effects of three glucocorticoid replacement regimens commonly administered in SAI patients on a broad range of psychological outcome parameters. We implemented a double-blinded, randomized, crossover design and additionally compared the main psychological outcome parameters to data from patients with pituitary disease and adrenal sufficiency as well as to an age- and sexmatched healthy control group.

\section{Materials and methods}

\section{Study population}

Medical records from $n=248$ patients who had undergone pituitary surgery at the University Hospital of Essen, Germany, and evaluation of the adrenal function within the previous 12 months were screened (see Fig. 1 for a diagram, which also includes information on exclusion and dropout). Inclusion criteria were age 18-75 years and stable substitution therapy of all pituitary axes (if necessary) for at least 3 months. Patients were excluded if Beck Depression Inventory score exceeded the cutoff indicating moderate-to-severe depressive symptoms (12). Pregnancy and a previous history of hypercortisolism also led to exclusion from the study. A peak cortisol of more than $500 \mathrm{nmol} / \mathrm{l}$ during the insulin tolerance test was used to categorize the patients as adrenal sufficient (patient controls (PC)) and a peak cortisol of $<450 \mathrm{nmol} / \mathrm{l}$ to diagnose SAI. Owing to difficulties in establishing a clear diagnosis, patients

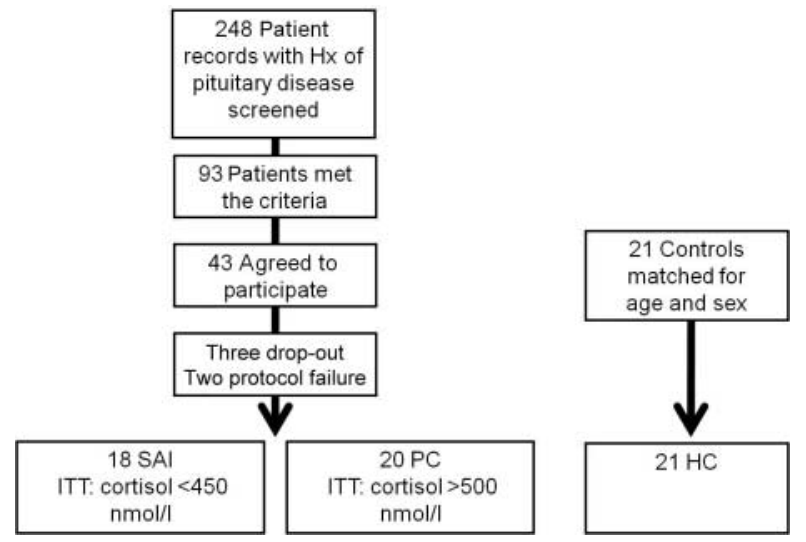

Figure 1 Patients $(n=248)$ with a history of pituitary surgery were screened. A peak cortisol of more than $500 \mathrm{nmol} / \mathrm{l}$ during the ITT was used to categorize patients as adrenal sufficient (patient controls (PC)) and a peak cortisol of $<450 \mathrm{nmol} / \mathrm{l}$ to diagnose secondary adrenal insufficiency (SAI). HC, hydrocortisone. with peak cortisol levels between 450 and $500 \mathrm{nmol} / \mathrm{l}$ were excluded from the study. Ninety-three patients met all inclusion criteria, of those $43(n=23$ SAI, $n=20 \mathrm{PC}$ ) agreed to participate. Three SAI patients dropped out after initial consent but before treatment, and two SAI patients were excluded during the study for noncompliance, resulting in $18 \mathrm{SAI}$ patients and 20 PC that completed the study protocol.

Before enrollment in the study, 12 SAI patients $(66.6 \%)$ received a replacement regimen identical to treatment $A$, three patients $(16.7 \%)$ received replacement identical to treatment B (for replacement therapies in this study, see below), and three patients (16.6\%) received different regimens (i.e. 7.5-5-0, 20-0-0, and 15-10-0 mg). The average dose of HC before study entry was $17.1 \pm 4.3 \mathrm{mg}$. SAI patients received their original replacement regimen after they had completed the study, as data analysis and interpretation were ongoing at this time point. All patients with thyrotropic insufficiency received appropriate substitution with L-thyroxine and all patients with gonadotropic insufficiency received replacement with sex hormones except for postmenopausal women. Patients with somatotropic insufficiency received GH depending on clinical symptoms. SAI patients had significantly longer disease duration $(P<0.05)$ and significantly more impairments of other pituitary axes (i.e. thyreotropic, gonadotropic, and somatotropic axis) compared with PC. For detailed information regarding clinical characteristics of SAI and PC, see Table 1 . Healthy controls $(n=21)$ were recruited by public advertisement and matched to SAI group by age and sex.

\section{Study protocol}

The study was approved by the Local Ethics Committee (permit no. 03-2279) and written informed consent was obtained from all participants. Based on a randomized, double-blind, crossover study design, each SAI patient received three different established glucocorticoid replacement therapies (i.e. treatment A, hydrocortisone $10 \mathrm{mg}$-placebo-5 mg-placebo; treatment $\mathrm{B}$, hydrocortisone $10 \mathrm{mg}-5 \mathrm{mg}$-placebo-5 mg; and treatment $\mathrm{C}$, prednisone $5 \mathrm{mg}$-placebo-placeboplacebo) for 4-week periods. The order of replacement schemes was randomized to control for sequence effects and blinded for patients and study personnel. Identically looking capsules containing either medication or placebo were prepared by the pharmacy of the University Hospital Essen. Capsules were designed to be completely resolved within $30 \mathrm{~min}$; hence, an effect of capsules on the pharmacokinetics of the active drugs can be excluded. Capsules were administered at distinct time points (i.e. 0700, 1200, 1500, and 1800 h). Given that a wash out period is not feasible in adrenal insufficient patients, questionnaires were completed at the end of each 4-week treatment regimen. At the end of each treatment period, patients returned to the study 
Table 1 Clinical characteristics of patients with SAI and PC.

\begin{tabular}{|c|c|c|c|}
\hline & SAI $(n=18)$ & PC $(n=20)$ & $\boldsymbol{P}$ \\
\hline Duration of disease in years (median (25th/75th quartile)) & $3.0(1.0 / 7.7)$ & $2.0(1.0 / 3.7)$ & $<0.021$ \\
\hline Time since resection in years (median (25th/75th quartile)) & $2.6(1.1 / 11.5)$ & $2.0(0.5 / 2.5)$ & 0.13 \\
\hline Thyreotrope insufficient $(\%(n))$ & $77.8(14)$ & $45(9)$ & $<0.041$ \\
\hline Gonadotrope insufficient $(\%(n))$ & $88.9(16)$ & $15(3)$ & $<0.001$ \\
\hline Somatotrope insufficient $(\%(n))$ & $83.3(15)$ & $20(4)$ & $<0.001$ \\
\hline With GH-substitution $(\%(n))$ & $46.7(7 / 15)$ & $75.0(3 / 4)$ & \\
\hline Diabetes insipidus $(\%(n))$ & $27.8(5)$ & $10.0(2)$ & 0.22 \\
\hline Pituitary surgery $(\%(n))$ & $88.9(16)$ & $90.0(18)$ & 0.66 \\
\hline Number of resections & & & 0.15 \\
\hline No resection $(\%(n))$ & $11.1(2)$ & $10.0(2)$ & \\
\hline One resection $(\%(n))$ & $61.1(11)$ & $85.0(17)$ & \\
\hline Two or more resections $(\%(n))$ & $27.8(5)$ & $5(1)$ & \\
\hline Transcranial resection $(\%(n))$ & $33.3(6)$ & $21.1(4 / 19)$ & 0.32 \\
\hline Underlying disease $(\%(n))$ & & & 0.34 \\
\hline NFA & $66.7(12)$ & $50.0(10)$ & \\
\hline Acromegaly & $0.0(0)$ & $20.0(4)$ & \\
\hline Prolactinoma & $5.6(1)$ & $5.0(1)$ & \\
\hline Hypophysitis & $5.6(1)$ & $0.0(0)$ & \\
\hline Congenital panhypopituitarism & $5.6(1)$ & $0.0(0)$ & \\
\hline Dysgerminoma & $5.6(1)$ & $0.0(0)$ & \\
\hline Astrocytoma & $0.0(0)$ & $5.0(1)$ & \\
\hline Rathke cyst & $0.0(0)$ & $5.0(1)$ & \\
\hline Craniopharyngioma & $11.1(2)$ & $10.0(2)$ & \\
\hline Meningioma & $0.0(0)$ & $5.0(1)$ & \\
\hline
\end{tabular}

NFA, nonfunctional adenoma; SAI, secondary adrenal insufficiency; PC, patient control. Significant group differences are printed in bold.

center to submit the completed questionnaires and to receive the next drug supply, lasting for 4 weeks, together with precise instructions on how to administer the capsules. Patient and healthy controls were assessed for only one 4-week period.

\section{Questionnaires}

The effects of the different treatment regimens were assessed using validated questionnaires, described in detail below. On the last day of each 4-week treatment block (i.e. Sunday at $1200 \mathrm{~h}$ ), quality of life (SF-36) and emotional distress (Brief symptom inventory( BSI)) were assessed retrospectively. Additionally, current psychological well-being (Bf-S) and alertness (Stanford Sleepiness Scale (SSS)) were measured over the course of the same day at 0700, 1500, 1800, and $2200 \mathrm{~h}$.

i) Health-related quality of life was assessed using the German version of the 36-item short-form health survey (SF-36), which quantifies various aspects of health-related quality of life in the last 4 weeks. The SF-36 contains eight subscales, which may be combined into two global health measures, i.e. the SF-36 Physical Sum score and the SF-36 Psychological Sum score, with lower scores indicating impaired quality of life (13). This generic instrument was used given the lack of validated disease-specific instruments translated into German.

ii) The German short version (BSI) (14) of the Symptom Check List 90 (SCL-90-R) was used to assess emotional distress during the past 4 weeks.
The BSI contains 53 items with a five-point scale (0, not at all; 4, extremely). The average score of all items yields the Global Severity Index, which represents the overall level of distress. Higher scores indicate higher distress.

iii) Current psychological well-being was assessed with the German Bf-S (15). This validated checklist consists of 28 dichotomous adjectives describing opposing positive and negative psychological states (e.g. alive, faint; irritable, placid). Participants are instructed to choose one of the two dichotomous adjectives, which describes their current situation most adequately, or to choose a neutral statement 'neither - nor'. For analyses, a sum score for all items is calculated by assigning a ' 0 ' score for each positive statement, a ' 1 ' score for each neutral statement, and a ' 2 ', score for each negative statement. Hence, the Bf-S sum score is scaled from 0 to 56 with higher scores indicating impaired present state psychological well-being.

iv) The German version of the SSS (16) was used to assess alertness across the day. The SSS is widely used in the field of sleep and fatigue research and is composed of seven statements regarding current alertness or sleepiness respectively. A SSS score of 1 indicates maximum alertness, and a score of 7 is indicative of maximum sleepiness.

v) Overall satisfaction with the current medication was assessed within the SAI patient group using a $100 \mathrm{~mm}$ visual analog scale (from 'not at all' $(0 \mathrm{~mm})$ to 'very much' $(100 \mathrm{~mm}))$. 


\section{Statistical analyses}

i) Effects of replacement regimens on psychological parameters within SAI patients were assessed with repeated measures analysis of covariance (ANCOVA) controlling for disease duration. In case of significant ANCOVA treatment effects, post hoc paired $t$-tests were computed. For variables that were measured over the course of the study days (i.e. current well-being and alertness), two-way ANCOVAs with the repeated factors replacement treatment and time were computed.

ii) SAI patients were compared with patient and healthy controls with respect to sociodemographic and psychological parameters using independent samples $t$-tests, Mann-Whitney $U$ tests (in case of non-normally distributed variables) and Fisher's exact tests. For repeated measures, i.e. alertness and psychological well-being, ANOVAs with the factors time $\times$ group were calculated. In case of significant effects, post hoc t-tests to compare groups at different time points were conducted. Healthy controls were assessed for 1 month only; thus, separate analyses for the three replacement treatments were conducted. Normal distribution of data was evaluated using Kolmogorov-Smirnov test before data analysis. Results are presented as mean \pm s.D. if not otherwise indicated.

\section{Results}

\section{Sociodemographic characteristics}

Patients did not differ significantly from patient or healthy controls with respect to sociodemographic variables (Table 2).

\section{Quality of life and emotional distress}

Significant treatment effects were observed for the SF36 physical sum scale $(F=3.8, P<0.05$; Table 3$)$, and SF-36 subscales physical function $(F=3.8 ; P<0.05)$ and physical role function $(F=3.7, P<0.05)$ (Fig. 2). SAI patients reported improved physical quality of life (i.e. SF-36 physical sum scale, $P<0.05$; SF-36 subscale physical role function, $P<0.05)$ during treatment A (i.e. hydrocortisone $10 \mathrm{mg}$-placebo$5 \mathrm{mg}$-placebo) compared with treatment B (i.e. hydrocortisone $10 \mathrm{mg}-5 \mathrm{mg}$-placebo-5 mg). SF-36 physical function scores were significantly higher for treatment A vs treatment C (prednisone). No significant differences among treatment schemes were found for emotional distress (Table 3).

Compared with healthy controls, patients showed significantly impaired physical quality of life, i.e. lower scores on SF-36 physical sum scale (Table 3) as well as on most subscales, regardless of replacement regimen (all $P<0.05$; Fig. 2). Emotional distress was significantly higher in patients compared with healthy controls for all replacement treatments (all $P<0.05$; Table 3). No significant differences in quality of life and emotional distress between SAI patients and PC were observed (Table 3; Fig. 2).

\section{Current psychological well-being and alertness}

Significant changes in current well-being (Bf-S; Fig. 3) and alertness (SSS; Table 3) over the course of the day were observed irrespective of medication (all $P<0.05$, time effects), attributable to the circadian rhythm. Interestingly, a significant interaction effect (replacement regimen $\times$ time, $F=2.2, P<0.05$ ) was detected for current well-being (Bf-S). Post hoc analysis revealed a significant treatment effect at $1800 \mathrm{~h}(F=4.5$, $P<0.05)$, with impaired current well-being for treatment B (i.e. hydrocortisone $10 \mathrm{mg}-5 \mathrm{mg}$-placebo-5 mg) compared with treatment A (i.e. hydrocortisone $10 \mathrm{mg}-$ placebo-5 mg-placebo) $(P<0.05)$ and treatment $\mathrm{C}$ (i.e. prednisone $5 \mathrm{mg}$-placebo-placebo-placebo; $P<0.05$; Fig. 3). No further treatment effects were detected for current psychological well-being or alertness.

Compared with healthy controls, SAI patients displayed an impaired current well-being at 1200, 1500, 1800, and $2200 \mathrm{~h}$, independent of replacement treatments (all $P<0.05$; Fig. 2), whereas no differences were observable between SAI patients and PC (data not shown). Interestingly, alertness did not differ between SAI patients and HC (Table 3), as well as SAI patients and PC (data not shown) over the course of the day.

Table 2 Sociodemographic characteristics of patients with SAI, PC, and HCs.

\begin{tabular}{|c|c|c|c|c|c|}
\hline & SAI $(n=18)$ & PC $(n=20)$ & $\boldsymbol{P}(\mathrm{SAl}$ vs $\mathrm{PC})$ & $\mathrm{HC}(n=21)$ & $\boldsymbol{P}(\mathrm{SAl}$ vs $\mathrm{HC})$ \\
\hline Age (mean \pm s.D.) & $52 \pm 10.3$ & $51 \pm 15.6$ & 0.89 & $53 \pm 14.0$ & 0.91 \\
\hline $\operatorname{Sex}(\%(n))$ & q: $5 \overline{5.6}(10)$ & ㅇ: 40.0 (8) & 0.52 & ㅇ: $4 \overline{2} .9(9)$ & 0.53 \\
\hline Married or with a partner $(\%(n))$ & $66.7(12)$ & $45.0(9)$ & 0.52 & $85.0(17 / 20)$ & 0.26 \\
\hline High school degree (German Abitur; \% $(n)$ ) & $29.4(5 / 17)$ & $26.3(5 / 19)$ & 0.84 & $20.0(4 / 20)$ & 0.70 \\
\hline Employment full time or part time $(\%(n))$ & $61.1(11)$ & $60.0(12)$ & 0.94 & $45.0(9 / 20)$ & 0.35 \\
\hline BMI (mean \pm s.D.) & $27.0 \pm 7.4$ & $30.6 \pm 5.5$ & 0.10 & $26.0 \pm 3.2$ & 0.58 \\
\hline
\end{tabular}

Secondary adrenal insufficiency (SAI) did not differ significantly from patient controls (PC) or healthy controls (HCs) respectively. 
Table 3 Quality of life (SF-36), emotional distress (BSI), and alertness (SSS) in patients with secondary adrenal insufficiency (SAI), patient controls (PC), and healthy controls (HC). Quality of life (SF-36 sum scores), emotional distress (BSI), alertness over the course of the day (Stanford Sleepiness Scale (SSS)), and satisfaction with medication were compared within SAI patients for different replacement regimens (i.e. treatment A, hydrocortisone $10 \mathrm{mg}$-placebo-5 mg-placebo; treatment $\mathrm{B}$, hydrocortisone $10 \mathrm{mg}-5 \mathrm{mg}-$ placebo-5 mg; and treatment $\mathrm{C}$, prednisone $5 \mathrm{mg}$-placebo-placebo-placebo).

\begin{tabular}{|c|c|c|c|c|c|}
\hline & \multirow[b]{2}{*}{$\mathrm{HC}(n=21)$} & \multirow[b]{2}{*}{ PC $(n=20)$} & \multicolumn{3}{|c|}{ SAl patients $(n=18)$} \\
\hline & & & Treatment A & Treatment B & Treatment C \\
\hline SF-36 physical sum scale & $53.0 \pm 10.4^{\dagger}$ & $45.8 \pm 11.7$ & $43.9 \pm 10.5^{\star}$ & $40.7 \pm 13.4^{\star}$ & $42.8 \pm 12.2$ \\
\hline SF-36 psychological sum scale & $52.6 \pm 9.2$ & $50.2 \pm 10.7$ & $46.3 \pm 8.7$ & $46.4 \pm 12.8$ & $46.5 \pm 12.7$ \\
\hline BSI Global Severity Index & $49.3 \pm 13.4^{\dagger}$ & $56.7 \pm 13.5$ & $57.9 \pm 11.4$ & $58.1 \pm 12.9$ & $58.2 \pm 13.5$ \\
\hline Stanford Sleepiness Scale score $(0700 \mathrm{~h})$ & $2.2 \pm 0.25$ & $2.5 \pm 0.35$ & $2.5 \pm 0.35$ & $2.3 \pm 0.32$ & $2.4 \pm 0.33$ \\
\hline Stanford Sleepiness Scale score $(1200 \mathrm{~h})$ & $1.3 \pm 0.21$ & $1.7 \pm 0.24$ & $1.7 \pm 0.24$ & $1.7 \pm 0.29$ & $1.7 \pm 0.19$ \\
\hline Stanford Sleepiness Scale score (1500 h) & $1.4 \pm 0.13$ & $1.8 \pm 0.27$ & $1.8 \pm 0.27$ & $1.8 \pm 0.24$ & $2.0 \pm 0.39$ \\
\hline Stanford Sleepiness Scale score $(1800 \mathrm{~h})$ & $1.5 \pm 0.17$ & $1.7 \pm 0.21$ & $1.7 \pm 0.21$ & $2.1 \pm 0.30$ & $1.8 \pm 0.27$ \\
\hline Stanford Sleepiness Scale score (2200 h) & $2.4 \pm 0.37$ & $2.8 \pm 0.39$ & $2.7 \pm 0.39$ & $3.4 \pm 0.50$ & $3.3 \pm 0.48$ \\
\hline $\begin{array}{l}\text { Satisfaction with medication ( } 100 \mathrm{~mm} \text { visual } \\
\text { analog scale) }\end{array}$ & - & - & $51.2 \pm 33.1$ & $56.6 \pm 27.3$ & $62.1 \pm 28.5$ \\
\hline
\end{tabular}

A significant treatment effect was observed for SF-36 physical sum scale within SAI patients ( $P<0.05$, result of post hoc paired $t$-test, for ANOVA results, see text). Additionally, group differences were computed between SAI patients and PC or HC respectively. Significant group differences between SAI patients and $\mathrm{HC}$ were found for all replacement regimens $\left({ }^{\dagger} P<0.05\right.$, results of independent samples $t$-tests). SAI patients did not differ from PC. Lower SF-36 scores indicate impaired quality of life, higher BSI score increased emotional distress, and higher SSS scores impaired alertness.

\section{Satisfaction with treatment regimens}

Patient satisfaction did not differ significantly between treatment regimens (Table 3), but large interindividual differences in treatment satisfaction (as indicated by large S.D.s) were observable.

\section{Discussion}

By implementing a randomized crossover study, we aimed to assess the effects of three standard replacement regimens commonly used in the treatment of SAI (i.e. HC twice or thrice a day, prednisone) on patients' subjective health. The crossover design allowed the assessment of treatment effects independent of interindividual differences regarding hormonal axes disturbances, additional replacement therapies, and disease pathogenesis. Our data indicate improvements in physical quality of life and current well-being in the early evening during replacement with hydrocortisone 10-0-5-0 mg. Nevertheless, except for alertness, none of the replacement regimens could completely restore psychological parameters to the level of healthy controls.

We analyzed a broad range of psychological parameters to detect possible beneficial effects of standard replacement regimens on subjective wellbeing in a carefully recruited cohort of SAI patients. However, in line with previous experimental studies in patients with primary and/or SAI $(5,6,10)$, the observed effects were either small (for physical quality of life) or nonsignificant (for psychological quality of life and emotional distress). Patient satisfaction with the medication did not differ between replacement regimens, irrespective of the observed differences in quality of life. Together with the observed large interindividual variation of satisfaction with treatment regimen, these findings further underscore the previous suggestion by Arlt (1) '(...) to let the patient experiment with different timings to find the most suitable regimen for his individual needs'.

We observed a slight improvement in quality of life during $\mathrm{HC}$ replacement twice daily. This is at odds with the hypothesis that a thrice-daily substitution might better mimic the physiological diurnal cortisol variation (1). However, our standard twice-daily replacement regimen is confounded with the HC dosage. Hence, one may speculate that the beneficial effect of a twice-daily application may be rather attributable to a lower total dose of $\mathrm{HC}$ (i.e. 15 vs $20 \mathrm{mg}$ ). This is complemented by results of previous cross-sectional studies, which indicated an association between higher HC doses and impaired subjective health status $(7,9)$. Unfortunately, our study design does not allow to clearly differentiate the effects of HC dosage and replacement regimen (i.e. twice or thrice daily). The observed beneficial effects of the twice daily $\mathrm{HC}$ regimen may also be due to the timing of the $5 \mathrm{mg}$ dose, i.e. $1200 \mathrm{~h}$ in treatment B vs $1500 \mathrm{~h}$ in treatment A. The pharmacokinetic properties of HC may have led to low cortisol levels in the afternoon when administered at $1200 \mathrm{~h}$, which may be avoided by administration at $1500 \mathrm{~h}$. Whereas the primary aim of this study was to analyze the effects of clinically relevant and commonly used standard replacement regimens, the design of future studies should take the pharmacokinetic effects of $\mathrm{HC}$ into account.

Interestingly, physical role functioning (as assessed with the respective SF-36 subscale) was affected by HC replacement regimen in a previous study (9) and our study, suggesting that this area of quality of life may 


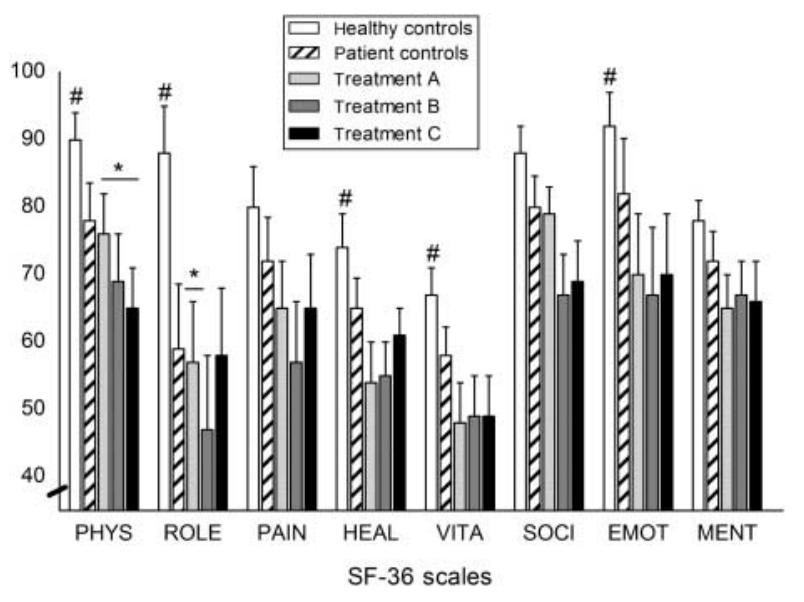

Figure 2 Health-related quality of life (SF-36) in patients with secondary adrenal insufficiency (SAI) for different replacement regimens (i.e. treatment $A$, hydrocortisone $10 \mathrm{mg}$-placebo- $5 \mathrm{mg}$ placebo; treatment $\mathrm{B}$, hydrocortisone $10 \mathrm{mg}-5 \mathrm{mg}$-placebo- $5 \mathrm{mg}$; and treatment $\mathrm{C}$, prednisone $5 \mathrm{mg}$-placebo-placebo-placebo) and in healthy controls $(\mathrm{HC})$. Higher scores indicate improved quality of life (SF-36). Physical role function was significantly improved during treatment $\mathrm{A}$ (i.e. hydrocortisone $10 \mathrm{mg}$-placebo-5 mg-placebo) compared with treatment $B$ (i.e. hydrocortisone $10 \mathrm{mg}-5 \mathrm{mg}$ placebo-5 mg), and physical function was improved during treatment $A$ vs treatment $C$ (prednisone) $\left({ }^{*} P<0.05\right.$, result of post hoc computed paired $t$-tests). Compared with $\mathrm{HC}$, SAl showed significantly impaired quality of life on most SF-36 subscales (i.e. PHYS, physical function; ROLE, physical role function; HEAL, general health perception; VITA, vitality; and EMOT, emotional role function), regardless of replacement regimen $\left({ }^{\#} P<0.05\right)$. No significant differences were observed for SF-36 subscales pain (PAIN), social role function (SOCI), and mental health (MENT). Patient controls did not differ significantly from SAl patients.

indeed be susceptible to different $\mathrm{HC}$ regimens. Importantly, we observed beneficial treatment effects only for physical aspects of quality of life, while psychological quality of life and emotional distress did not differ between replacement regimens. This leads to the conclusion that additional, e.g. supportive psychosocial approaches may be required to improve these psychological impairments.

The need for additional therapeutic approaches is further underlined by our data revealing marked impairments in subjective health in SAI patients compared with a matched healthy control group, independent of replacement regimen. This is consistent with previous observational studies comparing patients with primary AI and SAI to normative control groups $(2,7,8,9)$. One reason for impaired subjective health may be that common replacement regimens are not able to mimic the physiological diurnal pattern of cortisol secretion (1). This might be overcome by promising new developments of time-released medication $(17,18)$. In addition, subjective health may also be affected by multiple aspects of SAI such as the mere fact of having a chronic disease, the need for chronic medication, or working disability $(7,19)$. This is corroborated by our finding that patients from our control group (i.e. patients with pituitary disease and adrenal sufficiency) showed mutually the same impairments in psychological variables as the SAI patients, even though SAI patients also suffered from a more pronounced pituitary insufficiency. Nevertheless, given accumulating evidence that standard replacement regimens fail to completely restore quality of life in $\mathrm{AI}$ (1) and that patients with AI are at risk for severe affective disorders (3), clinicians should routinely screen their patients for psychiatric symptoms and/or psychological distress and may consider supportive psychosocial interventions $(19,20)$.

This study has limitations that should be considered when interpreting the results. The observed treatment effects for subscales (i.e. SF-36) or single time-points (i.e. SSS) should be cautiously interpreted in the light of small effect sizes and the potential risk of type-I-error, until further studies confirm these findings. Further, treatment outcome was assessed after 4-week periods, which are comparatively short for quality of life assessments. Longer treatment periods might have revealed (additional) treatment effects. Clearly, this study suffers from a lack of statistical power, which bears the risk of not being able to detect existing treatment effects (i.e. type II error), and may be one possible explanation why we did not observe differences in psychological variables between SAI patients and PC. However, SAI is not only a serious but also a relatively

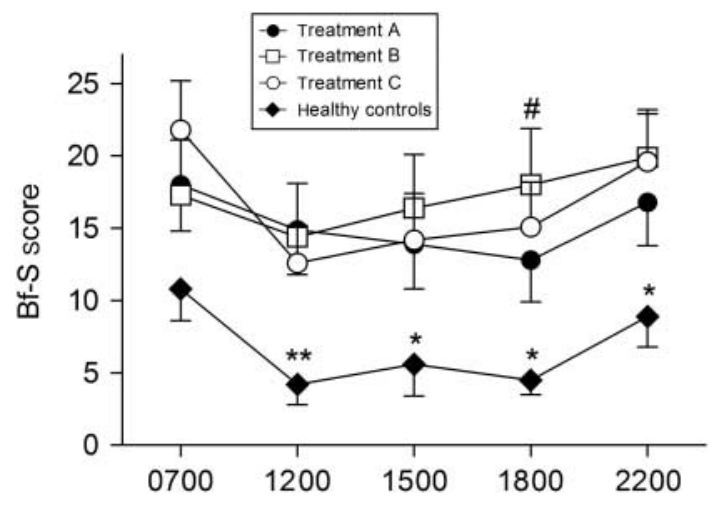

Figure 3 Current psychological well-being (Bf-S sum scores) in patients with secondary adrenal insufficiency (SAl) for different replacement regimens (i.e. treatment $A$, hydrocortisone $10 \mathrm{mg}$-placebo-5 mg-placebo; treatment $B$, hydrocortisone $10 \mathrm{mg}$ $5 \mathrm{mg}$-placebo- $5 \mathrm{mg}$; treatment C, prednisone $5 \mathrm{mg}$-placebo-placeboplacebo) and in healthy controls (HC). Higher scores indicate impaired psychological well-being (Bf-S). Significant changes of current wellbeing over the course of the day were observed independent of replacement regimen (all $P<0.05$, time effects) as well as for controls, attributable to circadian rhythm. Within SAI patients, a significant interaction effect (replacement treatment $\times$ time, $F=2.2, P<0.05$ ) was detected for current well-being (Bf-S). Post hoc tests indicated impaired current well-being at $1800 \mathrm{~h}$ during treatment $\mathrm{B}$ compared with treatment $A$ and treatment $C\left({ }^{\#} P<0.05\right)$. Compared with $\mathrm{HC}, \mathrm{SAl}$ patients displayed an impaired current well-being at 1200, 1500, 1800, and $2200 \mathrm{~h}$, independent of replacement treatment $\left({ }^{\star} P<0.05\right.$, ${ }^{\star \star} P<0.01$ vs each replacement scheme). 
rare disease. While this kind of study is urgently needed, it is extremely difficult to recruit larger patient cohorts, which is further underscored by the fact that our initial screening of $n=248$ patients who had undergone pituitary surgery resulted in a study cohort of only 18 patients. Keeping this restriction in mind, our data indicate beneficial effects of a 10-0-5-0 mg hydrocortisone replacement regimen on physical quality of life (i.e. physical functioning and physical role function) and current well-being in SAI patients. However, the effects were relatively small, and none of the treatments successfully restored subjective health when compared with healthy controls. These data underscore the need for more sophisticated physiological replacement regimens in combination with supportive psychosocial interventions in SAI patients.

\section{Declaration of interest}

The authors declare that there is no conflict of interest that could be perceived as prejudicing the impartiality of the research reported.

\section{Funding}

This research did not receive any specific grant from any funding agency in the public, commercial or not-for-profit sector.

\section{Author contribution statement}

$S$ Benson carried out statistical analyses and drafted the manuscript. P Neumann performed screening of medical records, data collection, and drafted the manuscript. $\mathrm{N}$ Unger performed screening of medical records, data collection, clinical examination, and characterization of patients. K Mann performed data collection, clinical examination, and characterization of patients. M Schedlowski conducted study design and supervision. S Elsenbruch conducted study design, carried out statistical analyses, and wrote final version of the manuscript. S Petersenn conducted study design, supervision, and wrote final version of the manuscript. All authors contributed to and have approved the final manuscript.

\section{References}

1 Arlt W. The approach to the adult with newly diagnosed adrenal insufficiency. Journal of Clinical Endocrinology and Metabolism 2009 94 1059-1067. (doi:10.1210/jc.2009-0032)

2 Lovas K, Loge JH \& Husebye ES. Subjective health status in Norwegian patients with Addison's disease. Clinical Endocrinology 200256 581-588. (doi:10.1046/j.1365-2265.2002.01466.x)

3 Thomsen AF, Kvist TK, Andersen PK \& Kessing LV. The risk of affective disorders in patients with adrenocortical insufficiency. Psychoneuroendocrinology 200631 614-622. (doi:10.1016/j.psy neuen.2006.01.003)

4 Arlt W, Rosenthal C, Hahner S \& Allolio B. Quality of glucocorticoid replacement in adrenal insufficiency: clinical assessment vs. timed serum cortisol measurement. Clinical Endocrinology 200664 384-389. (doi:10.1111/j.1365-2265.2006.02473.x)

5 Groves RW, Toms GC, Houghton BJ \& Monson JP. Corticosteroid replacement therapy: twice or thrice daily? Journal of the Royal Society of Medicine $1988 \mathbf{8 1} 514-516$.
6 Wichers M, Springer W, Bidlingmaier F \& Klingmuller D. The influence of hydrocortisone substitution on the quality of life and parameters of bone metabolism in patients with secondary hypocortisolism. Clinical Endocrinology $1996 \quad \mathbf{5 0} \quad 759-765$. (doi:10.1046/j.1365-2265.1999.00723.x)

7 Hahner S, Loeffler M, Fassnacht M, Weismann D, Koschker AC, Quinkler M, Decker O, Arlt W \& Allolio B. Impaired subjective health status in 256 patients with adrenal insufficiency on standard therapy based on cross-sectional analysis. Journal of Clinical Endocrinology and Metabolism $2007923912-3922$. (doi:10.1210/jc.2007-0685)

8 Bleicken B, Hahner S, Loeffler M, Ventz M, Allolio B \& Quinkler M. Impaired subjective health status in chronic adrenal insufficiency: impact of different glucocorticoid replacement regimens. European Journal of Endocrinology 2008159 811-817. (doi:10.1530/EJE08-0578)

9 Bleicken B, Hahner S, Loeffler M, Ventz M, Decker O, Allolio B \& Quinkler M. Influence of hydrocortisone dosage scheme on healthrelated quality of life in patients with adrenal insufficiency. Clinical Endocrinology $2010 \mathbf{7 2}$ 297-304. (doi:10.1111/j.1365-2265. 2009.03596.x)

10 Riedel M, Wiese A, Schurmeyer TH \& Brabant G. Quality of life in patients with Addison's disease: effects of different cortisol replacement modes. Experimental and Clinical Endocrinology 1993 101 106-111. (doi:10.1055/s-0029-1211215)

11 Alonso N, Granada ML, Lucas A, Salinas I, Reverter J, Oriol A \& Sanmarti A. Evaluation of two replacement regimens in primary adrenal insufficiency patients. Effect on clinical symptoms, healthrelated quality of life and biochemical parameters. Journal of Endocrinological Investigation 200427 449-454.

12 Hautzinger M, Bailer M, Worall H \& Keller F. Beck-DepressionsInventar (BDI). Bern: Hans Huber, 1995.

13 Bullinger M \& Kirchberger I. SF-36 Fragebogen zum Gesundheitszustand. Göttingen: Hogrefe Verlag, 1998.

14 Derogatis LR, Rickels K \& Rock AF. The SCL-90 and the MMPI: a step in the validation of a new self-report scale. British Journal of Psychiatry $1976 \mathbf{1 2 8}$ 280-289. (doi:10.1192/bjp. 128.3.280)

15 Zerssen D. Die Befindlichkeits-Skala - Bf-S. Weinheim: Beltz, 1976.

16 Glenville M \& Broughton R. Reliability of the Stanford Sleepiness Scale compared to short duration performance tests and the Wilkinson Auditory Vigilance Task. Advances in Bioscience 1978 21 235-244.

17 Debono M, Ghobadi C, Rostami-Hodjegan A, Huatan H, Campbell MJ, Newell-Price J, Darzy K, Merke DP, Arlt W \& Ross RJ. Modified-release hydrocortisone to provide circadian cortisol profiles. Journal of Clinical Endocrinology and Metabolism 200994 1548-1554. (doi:10.1210/jc.2008-2380)

18 Johannsson G, Bergthorsdottir R, Nilsson AG, Lennernas H, Hedner $\mathrm{T} \&$ Skrtic S. Improving glucocorticoid replacement therapy using a novel modified-release hydrocortisone tablet: a pharmacokinetic study. European Journal of Endocrinology 2009 161 119-123. (doi:10.1530/EJE-09-0170)

19 Baker SJK \& Wass JAH. Addison's disease. BMJ 2009339 b2384. (doi:10.1136/bmj.b2384)

20 Tiemensma J, Kaptein AA, Pereira AM, Smit JW, Romijn JA \& Biermasz NR. Coping strategies in patients after treatment for functioning or nonfunctioning pituitary adenomas. Journal of Clinical Endocrinology and Metabolism $201196 \quad 964-971$. (doi:10.1210/jc.2010-2490)

Received 25 April 2012

Revised version received 6 August 2012

Accepted 28 August 2012 